\title{
Sustaining remission in juvenile dermatomyositis
}

Sustained, complete remission of juvenile dermatomyositis (DM) could be achieved in just over 3 years of starting therapy using a protocol developed by researchers at The Children's Hospital Boston. Despite advances in therapy, patients with juvenile DM remain at significant risk of longterm mortality. "Our personal experience led to the development of a regimen to treat patients aggressively early in the course of the disease, with the goal of complete control of all detectable evidence of disease," explains lead investigator Susan Kim.

Patients presenting to Kim's institute with mild-to-moderate juvenile DM were treated immediately with three pulsed doses of intravenous methylprednisolone, followed by weekly pulsed-dose methylprednisolone, as well as methotrexate and daily prednisone; patients with mild disease received oral prednisone. Medications were tapered only when patients showed a complete clinical response (normalized muscle enzyme levels and muscle strength), whereas those who showed an insufficient treatment response after 3 months received additional medications including ciclosporin and intravenous immunoglobulin.

Of 49 patients observed over a 10-year period, 37 (69\%) achieved a complete clinical response at a median of 21 months and $28(57 \%)$ reached sustained remission at a median of 38 months. As well as supporting previous reports of successful treatment of juvenile DM, "these results suggest that our treatment approach leads to improved outcomes with higher rates of clinical response and remission than has previously been shown," says Kim.

Negin Nassabeh

Original article Kim, S. et al. Complete and sustained remission of juvenile dermatomyositis resulting from aggressive treatment. Arthritis Rheum. 60, 1825-1830 (2009). 